\title{
Erratum to: Pig lacks functional NLRC4 and NAIP genes
}

\author{
Chisato Sakuma $^{1,2}$ - Daisuke Toki ${ }^{3} \cdot$ Hiroki Shinkai ${ }^{1}$ Takato Takenouchi ${ }^{1}$. \\ Mitsuru Sato ${ }^{1} \cdot$ Hiroshi Kitani $^{1} \cdot$ Hirohide Uenishi ${ }^{1}$
}

Published online: 5 December 2016

(C) Springer-Verlag Berlin Heidelberg 2016

\section{Erratum to: Immunogenetics}

\section{DOI 10.1007/s00251-016-0955-5}

The article should have included the following statement of acknowledgement: 'While this paper was under review, a paper with a similar conclusion was published by Dawson and colleagues (Dawson et al. 2016)'.

\section{Reference}

Dawson HD, Smith AD, Chen C, Urban JF, Jr. (2016) Vet Microbiol 10.1016/j.vetmic.2016.05.013

The online version of the original article can be found at http://dx.doi. org/10.1007/s00251-016-0955-5.

\section{Hirohide Uenishi}

huenishi@affrc.go.jp

1 Animal Bioregulation Unit, Division of Animal Sciences, Institute of Agrobiological Sciences, National Agriculture and Food Research Organization, 1-2 Owashi, Tsukuba, Ibaraki 305-8634, Japan

2 Present address: National Institute of Infectious Diseases, 1-23-1 Toyama, Shinjuku, Tokyo 162-8640, Japan

3 Animal Research Division, Institute of Japan Association for Techno-innovation in Agriculture, Forestry and Fisheries, 446-1 Ippaizuka, Kamiyokoba, Tsukuba, Ibaraki 305-0854, Japan 